\title{
MicroRNA-374b-5p Functions as a Tumor Suppressor in Non-Small Cell Lung Cancer by Targeting FOXPI and Predicts Prognosis of Cancer Patients
}

This article was published in the following Dove Press journal: OncoTargets and Therapy

\author{
Jianzhao $\mathrm{Li}^{\prime}$ \\ Xinfang Zhang ${ }^{2}$ \\ Jiaying Tang ${ }^{3}$ \\ Cuixue Gong ${ }^{4}$ \\ 'Department of Pathology, Central \\ Hospital of Zibo, Zibo, Shandong 255000, \\ People's Republic of China; ${ }^{2}$ Clinical \\ Laboratory, Qilu Hospital Huantai \\ Branch, Zibo, Shandong 256400, People's \\ Republic of China; ${ }^{3}$ Department of Blood \\ Transfection, Central Hospital of Zibo, \\ Zibo, Shandong 255000, People's \\ Republic of China; ${ }^{4}$ Outpatient Dressing \\ Room, Central Hospital of Zibo, Zibo, \\ Shandong 255000, People's Republic of \\ China
}

Background: Lung cancer remains the most frequent malignancy worldwide with increasing morbidity and mortality. This study aimed to assess the expression of microRNA-374b-5p (miR$374 b-5 p$ ) in tissues and cell lines of non-small cell lung cancer (NSCLC) and to evaluate the prognostic value of miR-374b-5p as well as its biological function in tumor progression.

Materials and Methods: Expression of miR-374b-5p in NSCLC patients and cells was estimated using quantitative real-time PCR. The prognostic value of miR-374b-5p was evaluated using KaplanMeier method and Cox regression analysis. Gain-of-function and loss-of-function cell experiments were performed to examine the effects of miR-374b-5p on NSCLC cell proliferation, migration and invasion. A luciferase activity assay was used to confirm the target gene of miR-374b-5p.

Results: miR-374b-5p expression levels were decreased in tumorous tissues and cell lines compared with the normal tissues or cells $(P<0.05)$. The expression of miR-374b-5p was associated with the patients' tumor size, lymph node metastasis and TNM stage (all $P<$ 0.05). Patients with low miR-374b-5p expression have a shorter survival time (log-rank $P=$ 0.001 ), and the downregulated expression of miR-374b-5p was determined to be an independent prognostic indicator of NSCLC. In NSCLC cells, the overexpression of miR-374b$5 \mathrm{p}$ could inhibit NSCLC cell proliferation, migration and invasion and could directly target FOXP1.

Conclusion: This study found that the decreased miR-374b-5p predicts poor prognosis of NSCLC, and the upregulation of miR-374b-5p can inhibit NSCLC cell proliferation, migration and invasion. The data obtained from this study provide a novel candidate prognostic biomarker and a potential therapeutic target for NSCLC.

Keywords: non-small cell lung cancer, microRNA-374b-5p, prognosis, proliferation, migration, invasion

\section{Introduction}

Lung cancer remains the most frequent malignancy and the leading cause of cancerrelated mortality worldwide. ${ }^{1}$ Non-small cell lung cancer (NSCLC) is the most common subtype of lung cancer, accounting for approximately $85 \%$ of all lung cancer cases. $^{2}$ Although advances in diagnostic approaches, most of NSCLC patients are initially diagnosed with advanced tumors due to the lack of significant clinical manifestations. ${ }^{3}$ With the development in medical science and technology, a series of therapeutic strategies, including surgery, chemotherapy and radiotherapy, have been
Correspondence: Cuixue Gong Outpatient Dressing Room, Central Hospital of Zibo, No. 54 Gongqingtuan Road, Zibo, Shandong 255036, People's Republic of China

$\mathrm{Tel} / \mathrm{Fax}+86-533-2360149$

Email gongcx_731I@163.com 
developed, but the clinical outcomes in patients suffering from NSCLC remain dismal. ${ }^{4}$ Therefore, novel efficient therapeutic methods are urgently needed to improve the treatment of NSCLC.

MicroRNAs (miRNAs) are a group of small non-coding RNAs with no capacity for protein coding. ${ }^{5}$ They have important regulatory effects on the expression of genes by directly binding to the 3 '-untranslated regions (3'-UTR) of target messenger RNAs. ${ }^{6}$ In addition, the regulatory function of miRNAs has been uncovered in various cellular processes, such as cell proliferation, migration, differentiation and invasion. ${ }^{7,8}$ The role of miRNAs in human cancers has attracted increasing attention for their clinical significance and biological function. ${ }^{9}$ Emerging studies have reported that functional miRNAs could directly inhibit the expression of oncogenes or tumor suppressors, leading to the regulation in tumor progression. ${ }^{10,11}$ In NSCLC, some aberrant expressions of miRNAs have been identified and indicated to be functional molecules that involving in tumor pathogenesis, such as miR$1305^{12}$ and miR-133b. ${ }^{13}$ miR-374b-5p has been investigated in some human cancers, such as bladder cancer, ${ }^{14}$ pancreatic cancer ${ }^{15}$ and gastric cancer. ${ }^{16} \mathrm{~A}$ study by Xu et al has reported that miR-374b-5p expression was decreased in NSCLC cases by in silico analysis. ${ }^{17}$ However, the clinical significance and functional role of miR-374b-5p in NSCLC remain unclear.

To improve the treatment of NSCLC, this study sought to investigate the predictive value of miR-374b-5p in the prognosis of NSCLC patients by analyzing its expression patterns. The functional role of miR-374b-5p in NSCLC progression was assessed by assessing the effects of miR$374 b-5 p$ on tumor cell proliferation, migration and invasion. The results of this study might provide a novel candidate biomarker for NSCLC prognosis and a potential therapeutic target for NSCLC treatment.

\section{Materials and Methods}

\section{Patients and Tissue Collection}

One hundred and twenty-two paired tumorous and adjacent normal tissues were collected from 122 NSCLC patients, who underwent surgery at the Central Hospital of Zibo from 2011 to 2013. All the patients were histopathologically diagnosed with NSCLC. The patients received major resection of lung parenchyma (lobectomy, pneumonectomy) or sublobar resection (segmentectomy, wedge resection) due to impaired lung function, and the tumor tissues and noncancerous tissues were collected during the surgery and immediately stored in liquid nitrogen for RNA extraction. None of the patients received any anti-tumor therapy before sampling, and the clinicopathological characteristics of the patients were complete and recorded for subsequent analysis. The Tumor-NodeMetastasis (TNM) stage of the patients was determined by the criteria published by the American Joint Committee on Cancer Classification. ${ }^{18}$ All the patients were followed up for 5 years to obtain their survival information. The protocols of this study were approved by the Ethics Committee of Central Hospital of Zibo (approval No. HM-LC-2011-012), and each patient wrote the informed consent for the clinical sample and information use and analysis.

\section{Cell Culture and Transfection}

NSCLC cell lines A549, H460, H1299 and H2106 and a human normal bronchial epithelium cell line BEAS-2B were obtained from the Shanghai Cell Bank of Chinese Academy of Science (Shanghai, China). Cells were culture in the DMEM medium supplemented with 10\% FBS (Gibco, Thermo Fisher Scientific, Waltham, MA, USA) at $37^{\circ} \mathrm{C}$ in a humidified incubator with $5 \% \mathrm{CO}_{2}$.

To achieve the in vitro regulation of miR-374b-5p, miR-374b-5p mimic, miR-374b-5p inhibitor and their corresponding negative controls (mimic $\mathrm{NC}$ and inhibitor NC) were separately transfected into NSCLC cells using Lipofectamine 2000 (Invitrogen, Carlsbad, CA, USA) following the manufacturers' protocols.

\section{RNA Extraction and Quantitative Real-Time PCR (qRT-PCR)}

Total RNA, including miRNAs, was extracted from tissues and cells by TRIzol reagent (Invitrogen, Carlsbad, CA, USA). Single-stranded cDNA was synthesized from RNA using a PrimeScript RT reagent kit (TaKaRa, Shiga, Japan) following the instruction of the manufacturer. The SYBR green I Master Mix kit (Invitrogen, Carlsbad, CA, USA) on the 7500 RealTime PCR System (Applied Biosystems, USA) was used to perform qRT-PCR, with U6 and GAPDH as the internal controls. The relative expression of miR-374b-5p and forkhead box 1 (FOXP1) mRNA was calculated using the $2^{-\Delta \Delta \mathrm{Ct}}$ method.

\section{Cell Counting Kit (CCK)-8 Assay}

The cells obtained at $48 \mathrm{~h}$ post-transfection were seeded into 96-well plates (cell density of $5 \times 10^{3}$ ), and the cell proliferation was analyzed using a CCK- 8 assay. In brief, the cells were cultured for 3 days, and $10 \mu \mathrm{L}$ CCK- 8 solution was added at the time points of $0,24,48$ and $72 \mathrm{~h}$ followed by 4 
$\mathrm{h}$ of incubation. The absorbance of each well at $450 \mathrm{~nm}$ was measured using a microplate reader (Bio-Rad, USA).

\section{Transwell Assay}

NSCLC cell migration and invasion were analyzed using a Transwell assay. After $48 \mathrm{~h}$ of cell transfection, the cells (cell density of $5 \times 10^{4}$ ) with serum-free medium were added into the upper transwell chambers (Corning, USA). The lower chambers included cell culture medium added with 10\% FBS (Gibco, Thermo Fisher Scientific, Waltham, MA, USA). The chambers for migration assay had no Matrigel coating, but those for invasion assay were pre-coated with Matrigel (Corning, USA). After $24 \mathrm{~h}$ of incubation at $37^{\circ} \mathrm{C}$, the cells in lower chambers were stained and counted using a light microscope (Olympus Corporation, Tokyo, Japan).

\section{Luciferase Activity Assay}

The target genes of miR-374b-5p were predicted using TargetScan (http://www.targetscan.org/vert 72/), and FOXP1 was presumed as a potential target since it contained a complete sequence of miR-374b-5p at its $3^{\prime}-$-UTR. The wile type (WT) and the mutant type (MUT) 3'-UTR of FOXP1 were, respectively, cloned into the luciferase reporter vectors and were then co-transfected into A549 cells with miR-374b-5p mimic, miR-374b-5p inhibitor or the NCs by Lipofectamine 2000 (Invitrogen, Carlsbad, CA, USA) following the manufacturers' protocols. After $24 \mathrm{~h}$ of transfection, the luciferase activity in the cells was measured using a dual-luciferase reporter assay system (Promega, Madison, WI, USA).

\section{Statistical Analysis}

The statistical analyses were analyzed using SPSS 21.0 software (SPSS Inc., Chicago, IL) and GraphPad Prism 7.0 software (GraphPad Software, Inc., USA). All data were expressed as mean $\pm \mathrm{SD}$. Comparisons between groups were analyzed

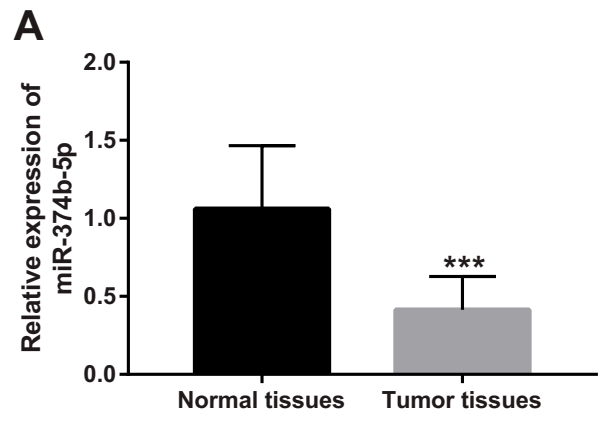

using Student's $t$-test or one-way ANOVA followed by Tukey's test. The relationship between miR-374b-5p and patients' clinical data was assessed using Chi-square test. Kaplan-Meier survival and Cox regression analyses were used to evaluate the prognostic value of miR-374b-5p in NSCLC patients. Pearson correlation analysis was used to assess the correlation of miR-374b-5p expression and FOXP1 mRNA levels in NSCLC patients. A result with a $P<0.05$ was indicated as statistically significant.

\section{Results}

\section{Expression of miR-374b-5p in NSCLC}

The expression of miR-374b-5p measured by qRT-PCR was found to be decreased in NSCLC tumor tissues compared with the adjacent normal tissues $(P<0.001$, Figure 1A). Similar results were found in NSCLC cells, which showed the downregulated expression of miR$374 b-5 p$ in the four NSCLC cell lines when compared to the normal cells (all $P<0.01$, Figure 1B).

\section{Association of miR-374b-5p with Clinicopathological Characteristics of NSCLC Patients}

The clinical features of NSCLC patients, including age, gender, tumor size, smoking history, differentiation, lymph node metastasis and TNM stage, are summarized in Table 1. To facilitate the subsequent analyses, NSCLC patients were divided into low and high miR-374b-5p expression groups based on the median miR-374b-5p expression value. By Chi-square test, the relationship between miR-374b-5p and the clinical features was assessed. The results indicated that the expression of miR374b-5p was associated with tumor size ( $P=0.018)$, lymph node metastasis $(P=0.001)$ and TNM stage $(P=0.002)$. No significant association was found between miR-374b-5p

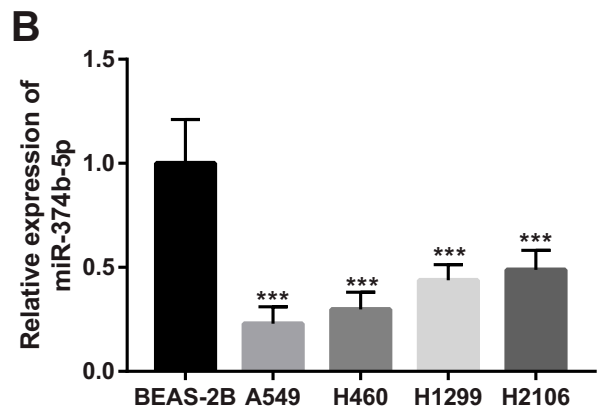

Figure I Expression of miR-374b-5p in NSCLC. (A) Expression of miR-374b-5p in the tissue samples collected from NSCLC patients. (B) Different expression levels of miR-374b-5p in four NSCLC cell lines. $* * * p<0.001$. 
Table I Association of miR-374b-5p and the Clinical Characteristics of the NSCLC Patients

\begin{tabular}{|c|c|c|c|c|}
\hline \multirow[t]{2}{*}{ Features } & \multirow{2}{*}{$\begin{array}{l}\text { Total No. } \\
n=122\end{array}$} & \multicolumn{2}{|c|}{ miR-374b-5p Expression } & \multirow[t]{2}{*}{$P$ values } \\
\hline & & $\begin{array}{l}\text { Low } \\
(n=64)\end{array}$ & $\begin{array}{l}\text { High } \\
(n=58)\end{array}$ & \\
\hline $\begin{array}{l}\text { Age (years) } \\
\quad \leq 60 \\
\quad>60\end{array}$ & $\begin{array}{l}44 \\
78\end{array}$ & $\begin{array}{l}24 \\
40\end{array}$ & $\begin{array}{l}20 \\
38\end{array}$ & 0.729 \\
\hline $\begin{array}{l}\text { Sex } \\
\text { Female } \\
\text { Male }\end{array}$ & $\begin{array}{l}50 \\
72\end{array}$ & $\begin{array}{l}26 \\
38\end{array}$ & $\begin{array}{l}24 \\
34\end{array}$ & 0.933 \\
\hline $\begin{array}{l}\text { Tumor Size }(\mathrm{cm}) \\
\qquad 3 \\
>3\end{array}$ & $\begin{array}{l}62 \\
60\end{array}$ & $\begin{array}{l}26 \\
38\end{array}$ & $\begin{array}{l}36 \\
22\end{array}$ & 0.018 \\
\hline $\begin{array}{l}\text { Smoking History } \\
\text { Never } \\
\text { Ever }\end{array}$ & $\begin{array}{l}47 \\
75\end{array}$ & $\begin{array}{l}25 \\
39\end{array}$ & $\begin{array}{l}22 \\
36\end{array}$ & 0.898 \\
\hline $\begin{array}{l}\text { Histological Type } \\
\text { Adenocarcinoma } \\
\text { Squamous cell } \\
\text { carcinoma } \\
\text { Others }\end{array}$ & $\begin{array}{l}48 \\
60 \\
14\end{array}$ & $\begin{array}{l}25 \\
29 \\
10\end{array}$ & $\begin{array}{l}23 \\
31 \\
4\end{array}$ & 0.296 \\
\hline $\begin{array}{l}\text { Differentiation } \\
\text { Well/Moderate } \\
\text { Poor }\end{array}$ & $\begin{array}{l}66 \\
56\end{array}$ & $\begin{array}{l}28 \\
36\end{array}$ & $\begin{array}{l}38 \\
20\end{array}$ & 0.016 \\
\hline $\begin{array}{l}\text { Lymph Node } \\
\text { Metastasis } \\
\text { Negative } \\
\text { Positive }\end{array}$ & $\begin{array}{l}65 \\
57\end{array}$ & $\begin{array}{l}25 \\
39\end{array}$ & $\begin{array}{l}40 \\
18\end{array}$ & 0.001 \\
\hline $\begin{array}{l}\text { TNM Stage } \\
\text { I-II } \\
\text { III-IV }\end{array}$ & $\begin{array}{l}58 \\
64\end{array}$ & $\begin{array}{l}22 \\
42\end{array}$ & $\begin{array}{l}36 \\
22\end{array}$ & 0.002 \\
\hline
\end{tabular}

and age, gender, smoking history, histological type and differentiation (all $P>0.05$ ).

\section{Clinical Significance of miR-374b-3p in}

\section{Prognosis of NSCLC}

This study recorded the survival information of NSCLC patients from a 5-year follow-up survey, and a survival curve was constructed using the Kaplan-Meier method. As shown in Figure 2, the median survival of NSCLC patients was 54 months, and this data was 45 months for patients with low miR-374b-5p expression. The survival curve data indicated that patients with low levels of miR-374b-5p had a shorter survival time compared with those with high levels of miR-374b-5p (log-rank $P=0.001$ ). The Cox regression analysis was subsequently performed, and the results listed in Table 2 indicated

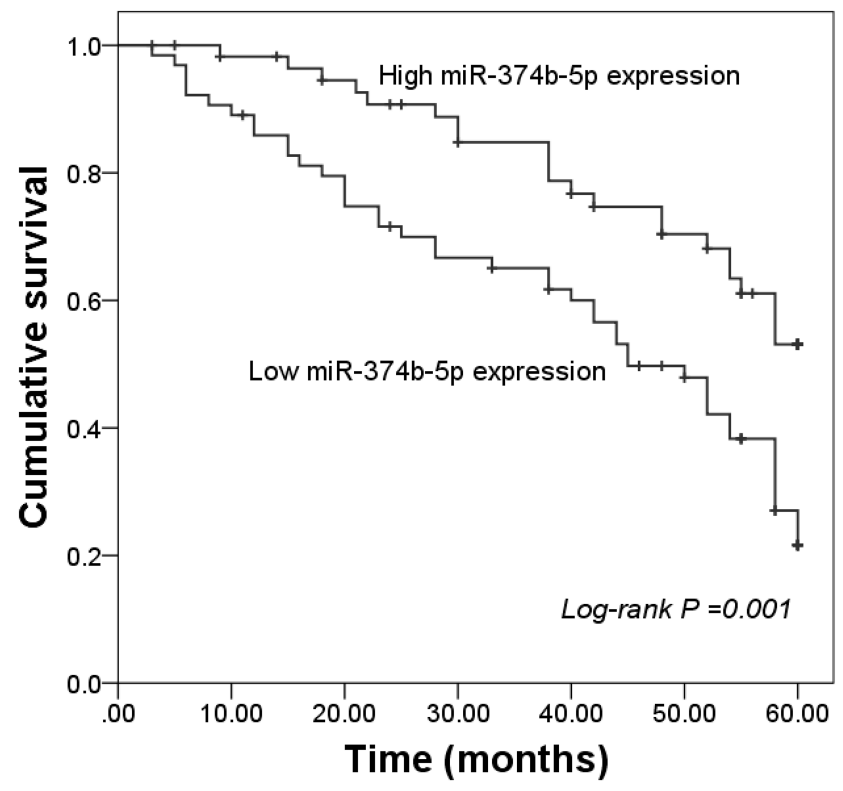

Figure 2 Kaplan-Meier survival curves for NSCLC patients based on different miR374b-5p expressions. Log-rank $P=0.001$.

that $\operatorname{miR}-374 \mathrm{~b}-5 \mathrm{p}(\mathrm{HR}=3.113,95 \% \mathrm{CI}=1.727-5.611$, $P<0.001)$ was an independent prognostic indicator for the survival of NSCLC patients.

\section{Inhibitory Effect of miR-374b-5p on NSCLC Cell Proliferation, Migration and Invasion}

To further understand the functional role of miR-374b-5p in the progression of NSCLC, the functional gain and loss experiments were performed in A549 and H460 cells. As shown in Figure 3A, the expression of miR-374b-5p was promoted by the miR-374b-5p mimic $(P<0.001)$, but was inhibited by the miR-374b-5p inhibitor $(P<0.001)$ in both the two NSCLC cell lines. The NSCLC cell proliferation was

Table 2 Cox Regression Analysis in the Patients with NSCLC

\begin{tabular}{|l|l|l|l|}
\hline \multirow{2}{*}{ Variables } & \multicolumn{3}{l|}{ Multivariate Analysis } \\
\cline { 2 - 4 } & HR & $\mathbf{9 5 \%} \mathbf{C l}$ & P value \\
\hline miR-374b-5p & 3.113 & $1.727-5.611$ & $<0.001$ \\
Age & 1.133 & $0.673-1.907$ & 0.638 \\
Gender & 1.042 & $0.627-1.731$ & 0.874 \\
Tumor size & 1.592 & $0.963-2.633$ & 0.070 \\
Smoking history & 1.094 & $0.633-1.893$ & 0.748 \\
Histological type & 1.131 & $0.823-1.845$ & 0.241 \\
Differentiation & 1.602 & $0.950-2.701$ & 0.077 \\
Lymph node metastasis & 1.621 & $0.937-2.805$ & 0.084 \\
TNM stage & 1.675 & $1.018-2.623$ & 0.049 \\
\hline
\end{tabular}


A

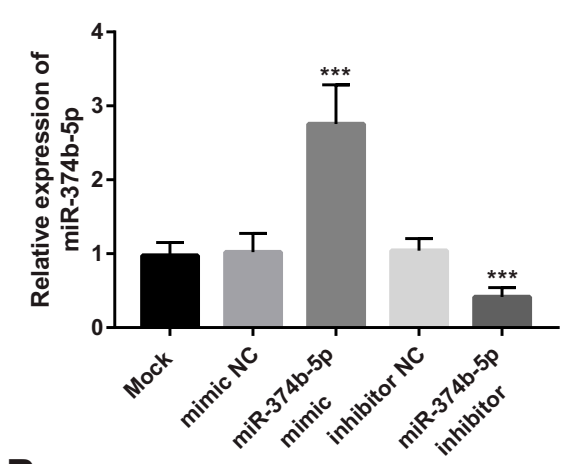

B

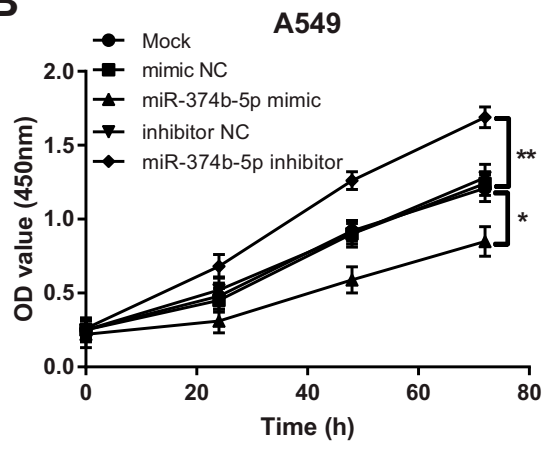

C

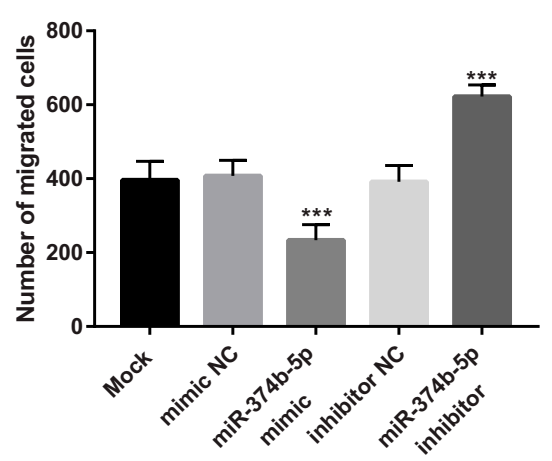

D

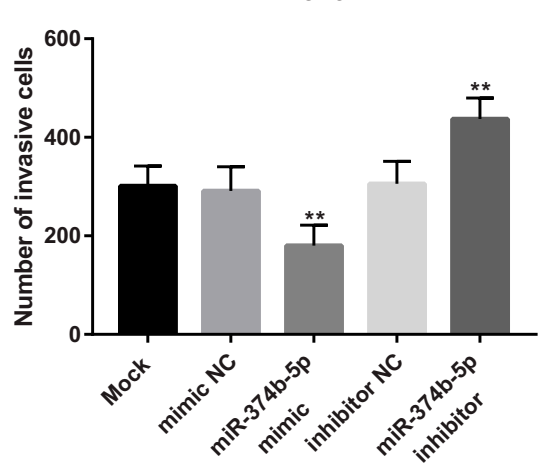

H460
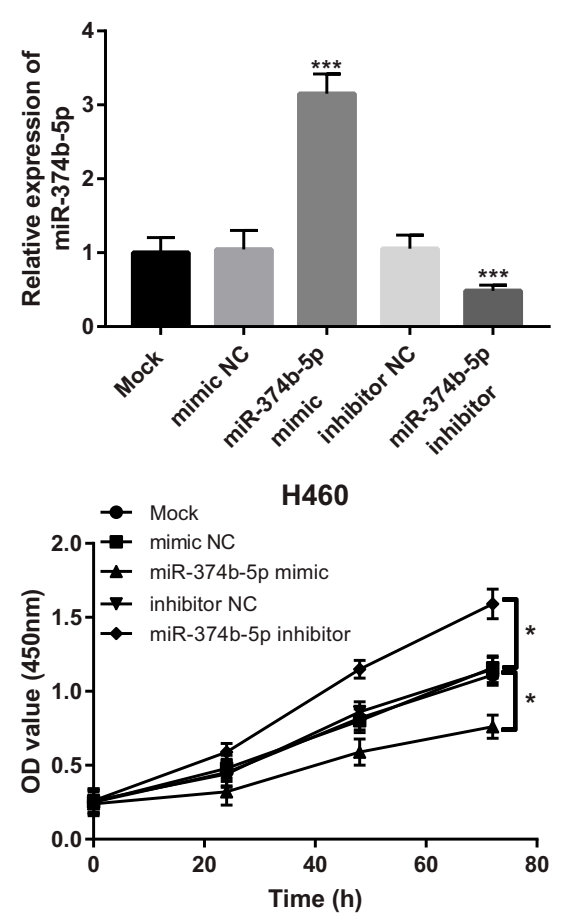

H460

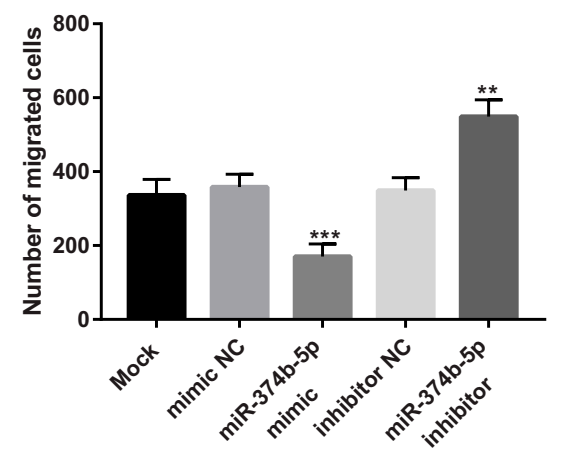

H460

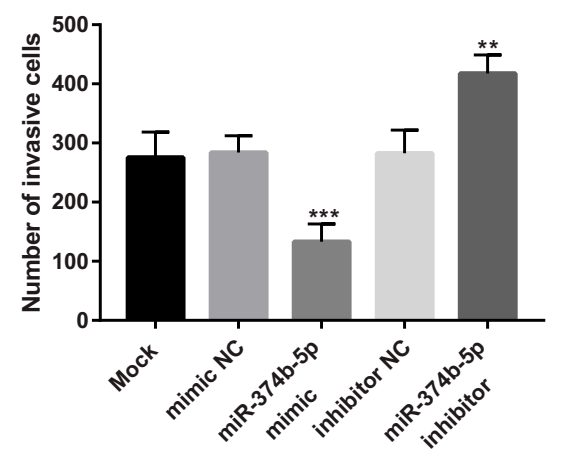

Figure 3 Effects of miR-374b-5p on cell proliferation, migration and invasion of A549 and H460 cells. (A) Cell transfection efficiency examination results. (B) Inhibiting effect of miR-374b-5p on NSCLC cell proliferation. (C and D) miR-374b-5p suppressed NSCLC cell migration (C) and invasion (D). $* P<0.05, * * p<0.0$ I, $* * * P<0.00$ I. 
significantly suppressed by the overexpression of miR-374b$5 \mathrm{p}$, while was enhanced by the knockdown of miR-374b-5p (all $P<0.05$, Figure 3B). The Transwell assay results showed that the upregulation of miR-374b-5p in NSCLC cells could inhibit, whereas the downregulation of miR-374b-5p could promote the cell migration and invasion (all $P<0.05$, Figure $3 \mathrm{C}$ and $\mathrm{D}$ ).

\section{FOXPI Serves a Target Gene of miR-374b-5p}

According to the bioinformatics prediction, the $3^{\prime}$-UTR of FOXP1 was found containing the complementary sequence of miR-374b-5p (Figure 4A). The following luciferase activity assay data shown in Figure 4B revealed that the luciferase activity of WT group was blocked by the overexpression of miR-374b-5p, but was enhanced by the reduction of miR374b-5p (both $P<0.05$ ). In the MUT group, no significant changes in luciferase activity were observed (all $P>0.05$ ). Furthermore, the mRNA expression of FOXP1 was as expected to be upregulated in NSCLC tissues $(P<0.001$, Figure 4C). A negative correlation was found between miR374b-5p expression and mRNA expression of FOXP1 in patients with NSCLC ( $\mathrm{r}=-0.841, P<0.001$, Figure 4D).

\section{Discussion}

Lung cancer is one of the most serious malignancies that threatens human life and health. Despite progresses in therapeutic strategies, the prognosis of NSCLC patients is still not ideal. Numerous miRNAs have been investigated in human malignancies with aberrant expression patterns during tumor progression. ${ }^{19,20}$ In NSCLC patients, expression of miR-141 was found to be increased in tumor tissues and cells, and the overexpression of miR-141 could promote tumor cell proliferation and invasion by targeting KLF9. ${ }^{21}$ miR-7-5p expression was reported to be downregulated in NSCLC tissues and suppressed tumor cell proliferation, migration and invasion through regulating NOVA2 expression. ${ }^{22}$ The decreased expression of miR-338 was also reported in NSCLC tissues and cell lines, and it could directly target NFATc1 to inhibit

\begin{tabular}{|c|c|}
\hline WT 3'-UTR of FOXP1 & $5^{\prime} \ldots$ GUUAAUAGGUGUUUUUUUUAUAC... \\
\hline has-miR-374b-5p & 3' GUGAAUCGUCCAACAUAAUAUA \\
\hline MUT 3'-UTR of FOXP1 & 5'...GUUAAUAGGUGUUUUAUAAUAUC... \\
\hline
\end{tabular}

C

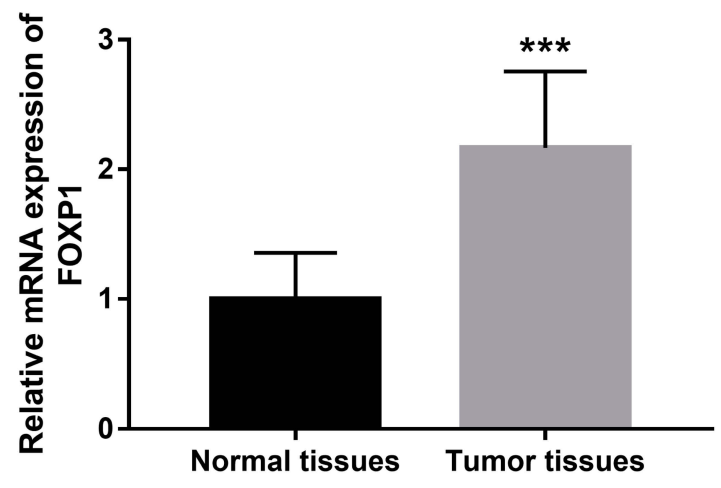

D
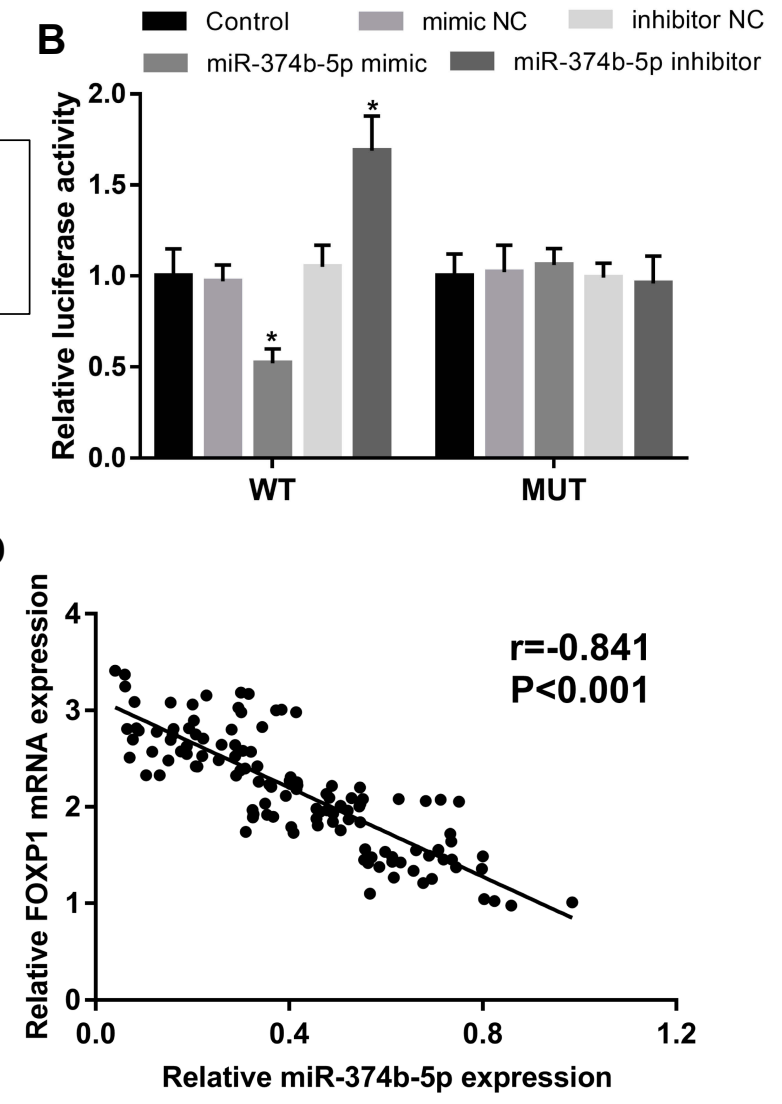

Figure 4 The prediction and confirmation of a target gene FOXPI of miR-374b-5p. (A) The complementary sequence of miR-374b-5p in the 3'-UTR of FOXPI. (B) Luciferase activity assay to verify the interaction between miR-374b-5p and FOXPI $(* P<0.05)$. (C) Relative mRNA expression of FOXPI in tissues collected from NSCLC patients $(* * * P<0.00 I)$. (D) Correlation between miR-374b-5p expression and FOXPI mRNA expression in NSCLC patients. 
cell proliferation of NSCLC cells. ${ }^{23}$ These published studies implied that miRNAs with aberrant expression patterns have important roles in the pathogenesis of human cancers. In the present study, we investigated the expression of miR-374b-5p in NSCLC tissues and cell lines. Xu et al have reported the decreased miR-374b-5p expression in NSCLC by in silico analysis, ${ }^{17}$ but no information was found in this study regarding the precise role of miR-374b-5p in NSCLC. According to the expression data obtained from our study, the expression of miR-374b-5p in NSCLC tissues and cell lines was downregulated relative to the normal tissues and cells, which is consistent with the published expression results. In addition, the association of miR-374b-5p with patients' tumor size, lymph node metastasis and TNM stage was further demonstrated. These results remind us to conclude that miR-374b-5p might be involved in the development of NSCLC.

The clinical outcomes of NSCLC patients remain dismal, especially in patients with advanced tumors. To improve the management of NSCLC patients, some deregulated miRNAs have been identified to be prognostic indicators to help to predict the clinical outcomes. For example, the increased expression of miR-421 in NSCLC tissues and serum samples has been indicated as an independent prognostic biomarker for NSCLC patients. ${ }^{24}$ The downregulated miR-133a in NSCLC patients was associated with prognosis of NSCLC. ${ }^{25}$ A recent study by Du et al also demonstrated that the decreased expression of miR-1298 served as a biomarker in NSCLC prognosis. ${ }^{26}$ Given the abnormal expression of miR-374b-5p in NSCLC tissues, the relationship between miR-374b-5p and the 5-year survival information was analyzed in NSCLC patients. The survival curves indicated that patients with low miR-374b-5p expression had a shorter survival time, and the Cox regression analysis revealed that miR-374b-5p was an independent prognostic indicator for patients with NSCLC. These data provided evidence for miR-374b-5p as a novel prognostic biomarker for NSCLC.

In recent decades, targeted cancer therapy has improved the clinical outcomes of patients with various human malignancies. ${ }^{27}$ The ability of miRNAs to regulate oncogenes or tumor suppressors leads them to be promising candidates for targeted therapy in NSCLC. Xie et al provided evidence for the important functional role of miR-29b, as indicated by the inhibiting effect of miR-29b on NSCLC cell proliferation, migration and invasion by targeting STRN4, which serves as an oncogene of NSCLC. ${ }^{28} \mathrm{Li}$ et al reported that the overexpression of miR-129 in NSCLC cells could inhibit cell proliferation, invasion and migration by directly regulating ZEB2, and might be a useful biomarker and therapeutic target of NSCLC. ${ }^{29}$ To further uncover the functional role of miR374b-5p in NSCLC progression, we regulated its expression in NSCLC cells and checked its effects on cell biological function. The overexpression of miR-374b-5p was found to inhibit NSCLC cell proliferation, migration and invasion, while the reduction of miR-374b-5p led to the opposite results, indicating that miR-374b-5p might serve as a tumor suppressive miRNA and a potential therapeutic target of NSCLC.

FOXP1 has been recognized as an oncogene in some human cancers, including ovarian cancer $^{30}$ and glioma. ${ }^{31}$ Of note, FOXP1 has been reported to mediate the promoting effects of some molecules in the progression of NSCLC. For example, LEF1-AS1 facilitated lung cancer cell proliferation and invasion by upregulating FOXP1. ${ }^{32}$ LINC01614 contributed to lung adenocarcinoma cell progression by increasing FOXP1. ${ }^{33}$ In the present study, a complementary sequence of miR-374b-5p has been found in the 3 '-UTR of FOXP1, and we demonstrated the interaction of miR-374b-5p with FOXP1 using a luciferase activity assay. In addition, a negative correlation was obtained between the expression of miR-374b-5p and FOXP1 mRNA expression in patients with NSCLC. The previously reported studies regarding the functional role of FOXP1 in lung cancer combined with our data inspired us to deduce that miR-374b-5p might be involved in the regulation of NSCLC progression by targeting FOXP1. The precise molecular mechanisms underlying miR-374b-5p in NSCLC progression were not deeply explored, which may be a limitation of this study, and warrant further investigations.

Taken together, all the data in this study indicated that the decreased miR-374b-5p in NSCLC patients is associated with poor prognosis, and the overexpression of miR-374b-5p can inhibit tumor cell proliferation, migration and invasion, indicating that miR-374b-5p may serve as a potential prognostic biomarker and a promising therapeutic target of NSCLC. This study provides a novel insight into the prognosis and treatment of NSCLC, and the methods to increase miR-374b-5p may be novel therapeutic strategies for NSCLC treatment.

\section{Author Contributions}

$\mathrm{JL}$ and CG made substantial contributions to conception and design, clinical analysis and interpretation of data, and was the major contributor in writing the manuscript. XZ and JT involved in cell experiments and data analysis. All authors contributed to data analysis, drafting or revising the article, 
gave final approval of the version to be published, and agree to be accountable for all aspects of the work.

\section{Disclosure}

The authors declare that they have no competing interests.

\section{References}

1. Hoffman RM, Sanchez R. Lung cancer screening. Med Clin North Am. 2017;101(4):769-785. doi:10.1016/j.mcna.2017.03.008

2. Peng W, Wang J, Shan B, et al. Diagnostic and prognostic potential of circulating long non-coding RNAs in non-small cell lung cancer. Cell Physiol Biochem. 2018;49(2):816-827. doi:10.1159/000493043

3. Nascimento AV, Bousbaa H, Ferreira D, Sarmento B. Non-small cell lung carcinoma: an overview on targeted therapy. Curr Drug Targets. 2015;16(13):1448-1463. doi:10.2174/1389450115666140528151649

4. Wang X, Zhang Y, Zhi X. Correlation between microRNA expression, clinicopathological characteristics, and prognosis in patients with non-small cell lung cancer: a retrospective study. Thorac Cancer. 2017;8(5):511-516. doi:10.1111/1759-7714.12480

5. Bhaskaran M, Mohan M. MicroRNAs: history, biogenesis, and their evolving role in animal development and disease. Vet Pathol. 2014;51 (4):759-774. doi:10.1177/0300985813502820

6. Ritchie W. microRNA target prediction. Methods Mol Biol. 2017;1513:193-200.

7. Liu S, Gao G, Yan D, et al. Effects of miR-145-5p through NRAS on the cell proliferation, apoptosis, migration, and invasion in melanoma by inhibiting MAPK and PI3K/AKT pathways. Cancer Med. 2017;6 (4):819-833. doi:10.1002/cam4.1030

8. Li Y, Sun W, Han N, Zou Y, Yin D. Curcumin inhibits proliferation, migration, invasion and promotes apoptosis of retinoblastoma cell lines through modulation of miR-99a and JAK/STAT pathway. $B M C$ Cancer. 2018;18(1):1230. doi:10.1186/s12885-018-5130-y

9. Qiu ZK, Liu N, Zhao SF, et al. MiR-1298 expression correlates with prognosis and inhibits cell proliferation and invasion of gastric cancer. Eur Rev Med Pharmacol Sci. 2018;22(6):1672-1679. doi:10.26355/eurrev_201803_14579

10. Mesci A, Huang X, Taeb S, et al. Targeting of CCBE1 by miR-330$3 \mathrm{p}$ in human breast cancer promotes metastasis. $\mathrm{Br} J$ Cancer. 2017;116(10):1350-1357. doi:10.1038/bjc.2017.105

11. Lang B, Zhao S. miR-486 functions as a tumor suppressor in esophageal cancer by targeting CDK4/BCAS2. Oncol Rep. 2018;39 (1):71-80. doi:10.3892/or.2017.6064

12. Cai Y, Hao Y, Ren H, et al. miR-1305 inhibits the progression of non-small cell lung cancer by regulating MDM2. Cancer Manag Res. 2019;11:9529-9540.

13. Chen GY, Ruan L. Downregulation of microRNA-133b and its clinical value in non-small cell lung cancer. Onco Targets Ther. 2019;12:9421-9434. doi:10.2147/OTT.S231312

14. Wang S, Zhang G, Zheng W, et al. MiR-454-3p and miR-374b-5p suppress migration and invasion of bladder cancer cells through targetting ZEB2. Biosci Rep. 2018;38(6). doi:10.1042/BSR20181436

15. Sun D, Wang X, Sui G, et al. Downregulation of miR-374b-5p promotes chemotherapeutic resistance in pancreatic cancer by upregulating multiple anti-apoptotic proteins. Int $J$ Oncol. 2018;52 (5):1491-1503. doi:10.3892/ijo.2018.4315

16. Xie J, Tan ZH, Tang X, et al. MiR-374b-5p suppresses RECK expression and promotes gastric cancer cell invasion and metastasis. World J Gastroenterol. 2014;20(46):17439-17447. doi:10.3748/wjg.v20.i46.17439
17. Xu C, Zheng Y, Lian D, et al. Analysis of microRNA expression profile identifies novel biomarkers for non-small cell lung cancer. Tumori. 2015;101(1):104-110. doi:10.5301/tj.5000224

18. Lim W, Ridge CA, Nicholson AG, Mirsadraee S. The 8(th) lung cancer TNM classification and clinical staging system: review of the changes and clinical implications. Quant Imaging Med Surg. 2018;8(7):709-718. doi:10.21037/qims.2018.08.02

19. Luo J, Liu L, Zhou N, et al. miR-519b-3p promotes responsiveness to preoperative chemoradiotherapy in rectal cancer patients by targeting ARID4B. Gene. 2018;655:84-90.

20. Tian L, Shan W, Zhang Y, et al. Up-regulation of miR-21 expression predicate advanced clinicopathological features and poor prognosis in patients with non-small cell lung cancer. Pathol Oncol Res. 2016;22 (1):161-167. doi:10.1007/s12253-015-9979-7

21. Kong Y-J, Tan -X-X, Zhang Y, et al. MiR-141 promotes cell proliferation and invasion in non-small cell lung cancer by targeting KLF9. Eur Rev Med Pharmacol Sci. 2019;23(23):10370-10378. doi:10.26355/eurrev 201912 19676

22. Xiao H. MiR-7-5p suppresses tumor metastasis of non-small cell lung cancer by targeting NOVA2. Cell Mol Biol Lett. 2019;24(1):60. doi:10.1186/s11658-019-0188-3

23. He W, Lu J. MiR-338 regulates NFATc1 expression and inhibits the proliferation and epithelial-mesenchymal transition of human non-small-cell lung cancer cells. Mol Genet Genom Med. 2020;8 (2):e1091. doi:10.1002/mgg3.1091

24. Li Y, Cui X, Li Y, Zhang T, Li S. Upregulated expression of miR-421 is associated with poor prognosis in non-small-cell lung cancer. Cancer Manag Res. 2018;10:2627-2633. doi:10.2147/CMAR.S167432

25. Shen Y, Chen F, Liang Y. MicroRNA-133a inhibits the proliferation of non-small cell lung cancer by targeting YES1. Oncol Lett. 2019;18 (6):6759-6765. doi:10.3892/ol.2019.11030

26. Du Z, Wu J, Wang J, et al. MicroRNA-1298 is downregulated in non-small cell lung cancer and suppresses tumor progression in tumor cells. Diagn Pathol. 2019;14(1):132. doi:10.1186/s13000-019-0911-4

27. Li J, Liang Y, Lv H, et al. miR-26a and miR-26b inhibit esophageal squamous cancer cell proliferation through suppression of c-MYC pathway. Gene. 2017;625:1-9. doi:10.1016/j.gene.2017.05.001

28. Xie Y, Zhao F, Zhang P, Duan P, Shen Y. miR-29b inhibits non-small cell lung cancer progression by targeting STRN4. Hum Cell. 2020;33 (1):220-231. doi:10.1007/s13577-019-00305-w

29. Li X, Li C, Bi H, et al. Targeting ZEB2 by microRNA-129 in non-small cell lung cancer suppresses cell proliferation, invasion and migration via regulating $\mathrm{Wnt} /$ beta-catenin signaling pathway and epithelial-mesenchymal transition. Onco Targets Ther. 2019;12:9165-9175. doi:10.2147/OTT.S217536

30. Choi EJ, Seo EJ, Kim DK, et al. FOXP1 functions as an oncogene in promoting cancer stem cell-like characteristics in ovarian cancer cells. Oncotarget. 2016;7(3):3506-3519. doi:10.18632/oncotarget.6510

31. Sun X, Wang J, Huang M, et al. STAT3 promotes tumour progression in glioma by inducing FOXP1 transcription. J Cell Mol Med. 2018;22 (11):5629-5638. doi:10.1111/jcmm.13837

32. Wang A, Zhao C, Gao Y, et al. LEF1-AS1 contributes to proliferation and invasion through regulating miR-544a/FOXP1 axis in lung cancer. Invest New Drugs. 2019;37(6):1127-1134. doi:10.1007/ s10637-018-00721-z

33. Liu AN, Qu HJ, Yu CY, Sun P. Knockdown of LINC01614 inhibits lung adenocarcinoma cell progression by up-regulating miR-217 and down-regulating FOXP1. J Cell Mol Med. 2018;22(9):4034-4044. doi: $10.1111 /$ jcmm. 13483 


\section{Publish your work in this journal}

OncoTargets and Therapy is an international, peer-reviewed, open access journal focusing on the pathological basis of all cancers, potential targets for therapy and treatment protocols employed to improve the management of cancer patients. The journal also focuses on the impact of management programs and new therapeutic

Submit your manuscript here: https://www.dovepress.com/oncotargets-and-therapy-journal agents and protocols on patient perspectives such as quality of life, adherence and satisfaction. The manuscript management system is completely online and includes a very quick and fair peer-review system, which is all easy to use. Visit http://www.dovepress.com/ testimonials.php to read real quotes from published authors. 